(1)

CrossMark

\title{
Making sense of telemedicine in the management of COPD
}

\author{
Jean Bourbeau and Raquel Farias
}

Affiliation: Respiratory Epidemiology and Clinical Research Unit, Research Institute of the McGill University Health Centre, McGill University, Montreal, QC, Canada.

Correspondence: Jean Bourbeau, Respiratory Epidemiology and Clinical Research Unit, Research Institute of the McGill University Health Centre, 5252 De Maisonneuve, room 3D.62, Montreal, QC, H4A 3S5, Canada. E-mail: jean.bourbeaudmcgill.ca

@ERSpublications

We should see telemedicine as an adjunct to self-management approaches, assisting proper healthcare coaching and helping patients to be more adherent in problem solving, decision making and acting earlier in the course of COPD exacerbations http://ow.ly/UNv530k2LcY

Cite this article as: Bourbeau J, Farias R. Making sense of telemedicine in the management of COPD. Eur Respir J 2018; 51: 1800851 [https://doi.org/10.1183/13993003.00851-2018].

Exacerbations of chronic obstructive pulmonary disease (COPD) are important events with negative impact on health status, hospitalisations and readmissions, and disease progression [1]. Treatments aim at reducing the risk of exacerbations and complications such as hospital admissions. Although treatment strategies to prevent COPD exacerbations and their subsequent hospital admissions are still largely based on pharmacological therapies, the Global Initiative for Chronic Obstructive Lung Disease 2017 also recommended non-pharmacological treatment, such as self-management interventions with communication with a healthcare professional [2]. The most recent Cochrane review reported that self-management interventions that include written action plans and coaching with a healthcare professional for a prompt treatment of worsening symptoms lead to a lower probability of both respiratory-related and all-cause hospitalisations, and also improve health status [3]. There remain problems with heterogeneity among interventions and consistency in their application that make generalisation difficult in real life. In light of these challenges, there has been increased interest in health information technology to ease the working life of health practitioners and to transform the way healthcare is delivered. There have been big promises made for the use of health information technology, with the rapid development of information and communication technologies for the management of chronic diseases. However, these promises may not have been realised in terms of tracking COPD patients for earlier detection of disease exacerbations and early intervention to minimise the deterioration that so frequently results in the need for hospitalisations $[4,5]$.

In PROMETE II, a multicentre, randomised, 12-month trial described by ANCOCHEA et al. [6] in this issue of the European Respiratory Journal, a specific telemedicine system was compared with routine clinical practice in patients with severe COPD. The system includes a remote patient-monitoring platform with equipment provided for blood pressure, oxygen saturation, heart rate and spirometry measurement, which were actively measured by the patient at home as per instructions, while respiratory rate and oxygen adherence data were passively collected by a device connected to the oxygen feed from their main oxygen source. Patients took measurements at the same time each day, at rest and after having taken their prescribed medication and with the oxygen therapy. The information was received by the monitoring centre, which used a triage application to grade the measurements into a traffic light system according to severity: red "clinical alert", i.e. one or more measurements exceeded the pre-established limits; yellow 
"technical alert", i.e. measurements were missing either through not being performed or not being received, which the application could escalate into a red "clinical alert"; and green, i.e. the measurements had been made and found to be within the predefined acceptable limits. This specific remote patient management system did not reduce COPD-related emergency department visits or hospital admissions compared with routine clinical practice. This study adds to the uncertainty about the role of telemedicine in COPD patient management.

Let's not throw the baby out with the bathwater. There is no choice but to move forward and adopt the technologies that power and connect our society within our healthcare system. The term telehealth includes a broad range of technologies and services to provide patient care and to improve the healthcare delivery system as a whole [7]. Telemedicine is a subset of telehealth that refers solely to the provision of healthcare services and education over a distance, through the use of telemonitoring and telecommunications technology [7]. There is evidence of improved clinician performance when information technologies are used in practice, including computer-assisted diagnosis, computerised reminders for preventive care, disease management systems, and computerised drug dosing and prescribing [8]. Telehealth could support new ways of delivering health services; for example, identifying patients across a large volume of records that experienced adverse drug events and examining the cause of the adverse event to create risk awareness programmes that could avoid these adverse events in the future $[9,10]$.

Telemedicine, both its application and results, is still controversial in COPD for a number of reasons. There has been substantial variability in the different telemonitoring interventions and applications used for the management of COPD. These have included recording and electronic transmission of physiological parameters and vital signs (spirometry, pulse oximetry, heart rate, breathing frequency and blood pressure); technology platforms for delivery of education and transmission of pedometer results; hand-held monitors, self-reported symptoms, and manually entered body temperature and oximetry; sensor-containing wrist-bands for heart rate, physical activity, near body temperature, and galvanic skin response; commercial oximeters and cell phones coupled with a wristband; self-reported data transmitted through cell phones (the EXACT-PRO (Exacerbations of Chronic Pulmonary Disease Tool Patient-Oriented Outcome) questionnaire); and automated alert calls based on winter weather conditions $[11,12]$. The variability among the telemonitoring applications precludes accurate comparison between studies. Therefore, the role of telemonitoring for overseeing patients and for enabling healthcare professionals to promptly intervene in the setting of an acute exacerbation is still controversial [5]. Monitoring of physiological parameters, as assessed in PROMETE II, does not solve the problem of predicting exacerbations that could lead to early therapy and prevention of hospital admissions. Recently, another study with telemonitoring and negative results has been published, the CHROMED trial [13]. The novel feature of monitoring forced oscillation technique compared with usual care in the CHROMED trial had no effect on time to first hospitalisation or quality of life. These recent studies add to the evidence that telemonitoring alone will be unlikely to have a major impact.

It is not sufficient to say that we must embrace the technologies that other sectors of the economy have successfully used to enhance efficiency and improve service. We should be giving more attention to the research we are doing and to the way that research in this specific area of early recognition and prompt treatment of exacerbations is being planned. First, let's put the necessary investments not solely in technology but in research that will answer the right questions. Can telemedicine: 1) improve self-management effectiveness by its availability at home, at the convenience of the patient, with the provision of information when it is needed most; 2) allow the patient to receive information that is specifically pertinent to their own condition and communicate with a care provider to receive support when they need it; 3) allow the healthcare professional to provide quality monitoring and support to a larger number of patients, while reserving more timely face-to-face interactions for patients who need it most? Too many research projects compare telemedicine with enhanced patient care management and coordination to usual care, i.e. traditional care delivery. This study design does not allow assessment of the real contribution of telemedicine as part of a complex multicomponent integrated care intervention, which draws heavily from the chronic care model [14]. Second, let's look more carefully at novel telemonitoring approaches that could serve patients' needs. A better understanding of respiratory physiology for early detection of COPD exacerbations should drive the agenda of researchers and those in the field of technology discovery. In addition, we need to think more of COPD as a heterogeneous and multidimensional disease. One novelty could be to integrate the remote monitoring of COPD-related comorbidities (such as diabetes and heart failure) that are also known to drive hospital readmissions. Third, faced with the competing budget priorities of immediate healthcare needs in our emergency rooms and hospitals, let's ensure that telemedicine and telehealth costs in COPD offer a real return on investments. Health economic studies are important to provide information to decision makers for 
efficient use of expensive telehealth resources for maximising health benefits. We cannot emphasise enough the importance of health economics in a world of proportionally increasingly scarce resources.

What should we expect from the use of telemonitoring and telecommunication technology in the management of COPD, in particular preventing complications of exacerbations? It may be more valuable to build on what we already know. Prompt recognition of worsening symptoms by the patient might be more sensitive than vital signs or physiological measures to ensure early detection and treatment of exacerbations. Most of the physiological changes and vital signs such as spirometry, pulse oximetry, heart rate and breathing frequency probably reflect an exacerbation being well established and/or complicated as opposed to an exacerbation being predicted or in an early stage. Instead, we need to use telehealth technologies to our advantage to increase patient activation and empowerment and to facilitate collaboration between patients and healthcare professionals. There is a large volume of evidence that supports self-management programmes in COPD [3,4], but we clearly need a more effective way to implement it. We need to standardise the way symptoms are being monitored and recorded by patients and healthcare professionals. Patients also need help to acquire the knowledge and skills required to follow medical therapies and adherence to health behaviour changes required to achieve optimal outcomes. We should see telemedicine as an adjunct to self-management approaches, assisting proper healthcare coaching and helping patients to be more adherent in problem solving, decision making and acting earlier in the course of COPD exacerbations.

Conflict of interest: J. Bourbeau reports grants from the Canadian Institute of Health Research (CIHR), Canadian Respiratory Research Network (CRRN) and the Research Institute of the MUHC and the FRQS; grants and personal fees (advisor and lectures) from AstraZeneca, Boehringer Ingelheim, GlaxoSmithKline, Grifols and Novartis, outside the submitted work.

\section{References}

1 Wedzicha JA, Seemungal TA. COPD exacerbations: defining their cause and prevention. Lancet 2007; 370: 786-796.

2 Vogelmeier CF, Criner GJ, Martínez FJ, et al. Global strategy for the diagnosis, management, and prevention of chronic obstructive lung disease 2017 report: GOLD executive summary. Eur Respir J 2017; 49: 1700214.

3 Lenferink A, Brusse-Keizer M, van der Valk PD, et al. Self-management interventions including action plans for exacerbations versus usual care in patients with chronic obstructive pulmonary disease. Cochrane Database Syst Rev 2017; 8: CD011682.

4 Criner GJ, Bourbeau J, Diekemper RL, et al. Prevention of acute exacerbations of COPD: American College of Chest Physicians and Canadian Thoracic Society Guideline. Chest 2015; 147: 894-942.

5 Pedone C, Lelli D. Systematic review of telemonitoring in COPD: an update. Pneumonol Alergol Pol 2015; 83: 476-484.

6 Ancochea J, García-Río F, Vázquez-Espinosa E, et al. Efficacy and costs of telehealth for the management of COPD: the PROMETE II trial. Eur Respir J 2018; 51: 1800354.

7 Kvedar J, Coye MJ, Everett W. Connected health: a review of technologies and strategies to improve patient care with telemedicine and telehealth. Health Aff (Millwood) 2014; 33: 194-199.

8 Garg AX, Adhikari NK, McDonald $\mathrm{H}$, et al. Effects of computerized clinical decision support systems on practitioner performance and patient outcomes: a systematic review. JAMA 2005; 293: 1223-1238.

9 Chaudhry B, Wang J, Wu S, et al. Systematic review: impact of health information technology on quality, efficiency, and costs of medical care. Ann Intern Med 2006; 144: 742-752.

10 Hripcsak G, Bakken S, Stetson PD, et al. Mining complex clinical data for patient safety research: a framework for event discovery. J Biomed Inform 2003; 36: 120-130.

11 McLean S, Nurmatov U, Liu JL, et al. Telehealthcare for chronic obstructive pulmonary disease: Cochrane Review and meta-analysis. Br J Gen Pract 2012; 62: e739-e749.

12 Vitacca M, Montini A, Comini L. How will telemedicine change clinical practice in chronic obstructive pulmonary disease? Ther Adv Respir Dis 2018; 12: 1753465818754778.

13 Walker PP, Pompilio PP, Zanaboni P, et al. Telemonitoring in COPD: the CHROMED study, a randomized clinical trial. Am J Respir Crit Care Med 2018; in press [https://doi.org/10.1164/rccm.201712-2404OC].

14 Tiedeman ME, Lookinland S. Traditional models of care delivery: what have we learned? J Nurs Adm 2004; 34 291-297. 